\title{
The Development and Application of Computer in Drug Analysis and Analytical Chemistry Education
}

\author{
Yuguang Lv*, Qi Shi \\ College of Pharmacy \\ Jiamusi University \\ Heilongjiang, Jiamusi 154007 \\ e-mail yuguanglv@163.com
}

\author{
Chunhui Shi \\ First affiliated hospital of Jiamusi University \\ Jiamusi, Heilongjiang, 154002, China \\ e-mail shichnhui66@163.com
}

\begin{abstract}
With the changing and development of the time, computers have been widely used in the pharmaceutical field in China. This paper mainly analyzes and summarizes the application of computers in the field of pharmacy. For example: The processing of experimental data, the analysis of drug structures, and many large-scale experimental devices need computer assistance. The combination of pharmacy and computer brings great convenience to the researchers. This paper introduces several technologies in the pharmaceutical field, and introduces the advantages of combining computer with these technologies. Through the aid of computers, it reflects the computer's ability to optimize various technologies, and it also shows that it can improve work efficiency. It also reflects the important role of computers in current society and the future development of science and technology, proves that computers have excellent development prospects and proves that the combination of computer and pharmacy has made progress in both are as.
\end{abstract}

\section{Keywords—Computer; Drug analysis; Analytical chemistry}

\section{INTRODUCTION}

The research directions of drug analysis include the study of drug stability, chemical and clinical tests, the utilization of drugs in biology, and the qualitative and quantitative analysis of active ingredients of Chinese herbs. Inspection and analysis of finished drugs, our grasp of quality during the production of drugs, regular inspection of quality when we store drugs, analysis of drugs in vivo and clinical drugs show drug analysis is extremely important for analytical chemistry. And drug analysis has a wide range of applications in the pharmaceutical field, and it has grown up to be an important branch of medicine now. At present, analysis and research of computers in pharmacy have made great progress. The combination of computer and pharmacy has made the field of pharmacy research more abundant.

\section{EASE OF USE}

\section{A. Computer Application in Atomic Absorption Spectroscopy}

In our analytical chemistry community, there is an extremely important research method, which is atomic

This study was financially supported by National Science Foundation of China (No. 21346006), Department of scientific research project in Heilong jiang province (No. B2017015), National project training project of Jiamusi University (No. JMSUJCGP 2016-003),. The leader of academic research and education, 2017 Jiamusi University education research hundred works. absorption spectroscopy (AAS).

Moreover, AAS has been applied in all areas of our lives, and it has played an active part in industrial metallurgy, scientific research, medicine and health, teaching, and agriculture. Then we study the application of computers in atomic absorption spectroscopy: AAS measures the content of elements through the resonance absorption generated by the characteristic radiation of the gaseous ground state atoms on the light source [1]. In AAS, the combination of a computer allows us to automate in the control of the instrument. For example, dropping samples, diluting samples, changing and replacing samples, and flushing can be automated. Makes the computer and the sample stage, the atomic absorption spectrometer and the printer form a manual operation control system. Another advantage is the fact that we can use the computer to view the state of the experiment and adjust it to the optimal state in time. When the working curve of the absorbance and concentration of the measured element is plotted, the coordinate scale can be converted by the computer. The computer can accumulate; average, measure and deduction base the measured signals. These can be automated. Then we can get the experimental results, and these results have been processed statistically[2].

\section{B. Application of computer in Gas Chromatography-Mass Spectrometry}

Mass spectrometry (MS) is the application of a magnetic field electric field to separate the moving ions according to their mass-to-charge ratio, and then to determine their molecular structure and determine the relative molecular mass. Gas chromatography (GC), as its name implies, is an analytical technique that sets the gas as the mobile phase and then performs separation analysis. The combination of the two can be used to identify the structure of a substance. For a compound that we do not even know what it is, applying this combination technique can accurately determine its relative molecular mass, and it can also measure the content of its different components. Measure the reaction principle and correct the wrong result. Therefore, the joint use technology has gained more and more attention [3]. The combination of a computer and this technology can also play an important role: Both the gas chromatograph and the mass spectrometer can be controlled by the computer at the same time, and the computer can also help us to count a large amount of complex data. When combined with a computer, the range of the scanning 
field is expanded, and the number of masses per second is close to 600. In the scanning process of the peak, the computer can also perform calculations quickly to determine the position and intensity of the peak. The combination of computer and GC-MS technology makes the data that we need to measure get without complex operation, making our experiment easier and simpler[4]. When using computer and GC-MS technology, the molecular structure of unknown substances can be judged by computer retrieval. Here's a detailed description: we can input the mass spectrum data of known substances into the computer. It's like we're building a mass spectrum database in a computer, and when we need to determine the structure of an unknown compound, we can compare it with the known data. Therefore we can extrapolate the structure of the compound. This approach is not applicable anywhere, and there are some new substances that are more difficult to determine structures in this way, so it is to continue to be refined and explored. The computer automates the instrument. The analog quantity measured by the instrument is converted to digital by an analog-to-digital conversion circuit, so that the computer can be processed more conveniently and the experimental data can be collected and counted. When we process the data, the computer can save us a lot of time, it can record data automatically, conversion the coordinate scale automatically, calibrate the instrument automatically and so on, we can do this experiment through the computer screen display requirements and reminders to carry out our experimental operation. Then we can use the machine to print the operation flow and the consequence of the operation [5]. GC-MS technology can be applied to the field of environmental science.The United States EPA uses this joint technology to determine the substances that pollute the atmosphere.

\section{Computer Application in Analytical Chemistry}

We can process the data through computers: we do experiments by using one element of statistics to process experimental data, and after many experiments, we count the data. In experiments related to analytical chemistry, if titration is required to determine the content of a substance, The linear equation between the concentration and the volume consumed in titration can be used to process the data, which is similar to the linear regression method. This method can make the experiment more convenient and the experimental data more accurate[6]. The computer can also help us predict the experimental conditions: we can let the computer borrow the equilibrium mechanism of the solution, rectify the side reaction coefficient, and show us accurate mathematical models. In this way, we can determine the experimental conditions of analytic chemistry. Here are a few examples: in color reaction, we can use this method to determine when the ph range is the best state. Calculate the amount of the flushing solution and the concentration of the flushing solution during the separation and purification by ion exchange. Sometimes when we encounter a more complex metal complex reaction, the computer can be used by us. We can use the computer to determine which ions are involved in the main reaction, which ions are not able to take part in the reaction, and we can get more reliable results by obtaining these conditions. The computer can help us to choose the best experimental conditions in the analytical chemistry experiment. The computer can help the experimenter accurately determine the composition of the substance accurately. We can use the equivalent model and the equilibrium model to eliminate unnecessary interference for our experiment. The equilibrium model means to get the balance of some chemical reactions through the equilibrium constant. And then we get a set of chemical equations. After we measure some unknown quantities through experiments, we can calculate the contents of the compounds under test and their coexistence interference elements together. When we deal with the problem of analytical chemistry, equilibrium models are often used, but sometimes the accuracy of equilibrium constants and the excessive concentration of the solution may have some effect on the use of the equilibrium model to analyze chemistry. The other model is based on the relationship between some of the conditional information(such as titration volume) and the content of the analyses before our experiment. By formulating these equations, we can calculate the data we need, for instance: the contents of each component. The equivalent model is more perfect than the equilibrium model technique. It can be used not only in chemical analysis, but also in instrumental analysis. Although it is more accurate than the equilibrium model, there are also bottlenecks in some cases.

\section{Application of Computer in Drug Analysis in vivo}

What we usually call in vivo drug analysis is to analyze and study the changes in the content of drugs in the body so as to obtain innumerable.

The parameters and transformations can also be used to understand the way medicines are metabolized in the body and the information in numerous places in the drug route. This type of analysis can be of great help in the areas of drug production, drug research, drug practice, and clinical testing. The improvement of drug efficacy and drug development can be of great help. Here are some of the characteristics of its research object: Low concentration; Low activity; complex sample components; Need for separation and purification; Less sample for analysis; Heavy workload requires us to submit the results quickly; Explaining the difficulties when dealing with data, etc. For these reasons, we often use gas chromatography, high performance liquid chromatography, ultraviolet analysis, fluorescence spectrometry, and instrumental analysis, in which instrumental analysis methods are often used. With the continuous development of technology, we have combined computer and instrument analysis methods to greatly improve the automation performance. After the dual use of technology, we can also optimize the conditions, optimize the experiment, and fit the curve [7]. In 1990, Wang Shunfu and Dong Youbo studied the topic of "computer program to calculate the in vitro release parameters of tablets", and propose a procedure for designing Weibull distribution parameters using the least-squares method that can be used in programming languages that are widely used in computers. The Weibull distribution parameter program is applicable to some frequently used minicomputers, such as the PC-1500. The experimental experience has proved that this kind of microcomputer program can make our operation more convenient, and the operation time is reduced much, and the result is very accurate, achieving the effect of a multiplier effect, and is more popular[8]. 


\section{E. The application of computer in chromatographic analysis and spectral analysis}

Both the chromatographic analysis and the spectral analysis results are accurate, and the analysis is fast, which is of eminent use in the field of drug analysis. T So far the most important method of separation and analysis has been chromatographic analysis. It is critical that we choose the chromatographic conditions, which is related to the success or failure of the experiment. High performance liquid chromatography (HPLC) has also been used extensively in drug analysis experiments. The factors that affect the analysis results are detectors, stationary phases, and mobile phases. Gao Shouguo used Access ST to create a database in the "Drug Chromatographic Separation Conditions Data System". The database management system was established with the language VB5. 0 introduced by Microsoft Corporation in February 1997. The VB language is appropriate for beginners. It is simple and straightforward, easy to operate, and can be associated with a lot of software. It provides convenience for us to review the data and can add or delete data. As well as updates and more. The chromatographic experimenter can reduce a lot of tedious work, and it can also provide us with great convenience in the selection of HPLC separation conditions when performing chromatographic analysis [9]. TLC technology can be said to be different from other technologies in the field of analytical research. Now it has also developed instrumentation, quantification, high efficiency, and computerization. We can use computers to cope with data problems encountered in the selection of conditions, saving a lot of time and manpower. We all know that the complexity of Chinese herbal medicines is extremely difficult to isolate and purify. We often use chromatographic and spectroscopic methods for the analysis of Chinese herbal medicines. These two methods are also used in conjunction with computers. So the computer provides an important role in the analysis of traditional Chinese medicine. Cluster analysis method is a relatively common data analysis method, which reflects the differences in different types of Chinese medicine, and the results of cluster analysis are very accurate and reliable, and have a great effect on the instrumentalization and computerization of traditional Chinese medicine identification [10]. .

\section{F. The Application and Prospect of computer in other areas}

In our field of drug analysis, if we want to make authoritative decisions on quality standards, it is extremely important to analyze the data. The workload is heavy. Because of our combination with the computer, much of our work has been automated and saved a lot of time, and the data is more accurate, more objective, and more comprehensive. Now, scientific research experts are working hard to perfect this technology and have made more and more remarkable achievements. In order to make computer research in the pharmacological field more in-depth, Chinese researchers are exploring and striving constantly, and have been paying attention. After this, our country has successively proposed "calculating drug analysis" and intends to make it become a discipline, and also proposed the physics of medicine analysis and calculations, etc. And proposed some suggestions for progress. These are all want to effectively combine the future progress of the pharmaceutical field and the computer field. Once, a scientist also proposed the concept of chemical statistics, which promoted mutual penetration and common development among various disciplines [11]. As China's technology in all aspects has been increasingly perfected, the combination of technologies in various aspects of our country has become ever more popular, so the combination of computer and pharmacy technology will be more and more perfect, pending development. Computers also have good applications in clinical testing. For example: Tang Jingbo, chief physician of Zhuhai, Guangdong, and others have jointly developed the "clinical medication monitor". This monitor is an important scientific tool for medical treatment. It can retrieve the interactions of hundreds of commonly used drugs, and what adverse reactions would happen when used together, and the $\mathrm{pH}$ value of various drugs, the use of drugs, and the use of dose between adults and children can automatically convert other functions. The medical and health unit is utilized widely. With it, our medical staff can more quickly and more clearly understand the interaction between drugs and drugs [12]. We all know that radiotherapy can treat cancers for people with malignancy. Then we can use a microcomputer to draw dose distribution curves more quickly, select the best solution for cancer patients, and improve the quality and efficiency of radiotherapy. Once, the United States put forward such a proposal: In order to formulate the best value of radiotherapy program to observe the radiation dose carefully, and then use a computer to show us radioactive information[13]. We can also use a computer to control drug delivery, which is a system that we also call the CAIMA SYSTEM. When the medical staff sets the standard of administration for the patient, the computer has a memory function that maintains this level of administration automatically. For example, an infusion monitor can control the speed and time of administration as well as the initial injection time for patients, which is a good treatment for some patients with diabetes [14]. This system has opened up a new world for the clinical testing community. We can use computers to ensure rational drug use. In the United States, many hospitals are used with computer equipment. In order to control drug delivery, satellite pharmacies in major wards will set up terminal equipment. Licensed pharmacists can send prescriptions given by doctors through CRTs and light pens. Into the system. This system has various functions. When we send it into the prescription, the system can show the dosage of medicine for our pharmacists and enable us to observe at any time. In the United States, clinics can also be online to automate patient-related information. The pharmacist can send data through the CRT, the system automatically shows us the dispensing information, automatic diagnosis, and some more perfect systems can also show us the best treatment plan, which can improve the efficiency of hospital treatment [15]. For example: Americans have used computers to reasonably calculate the dose of antibiotics used in patients with impaired renal function. The results of computer predictions are consistent with the values of our measured blood concentrations and avoid nephroptosis. There are so many applications of electronic computers in clinical testing, which proves that our country has paid great attention to the technology of computer and pharmaceuticals. 


\section{CONCLUSION}

Over the past few years, researchers in our country have continued to explore. The combination of computer and pharmacy has been increasingly perfected and continuously developed. Computers will infiltrate into the pharmaceutical field in the future and will be widely used by the medical community. Has become one of our indispensable technologies for cooperative use and has a very good prospect for development. This paper describes the application and analysis of the computer in the field of drug analysis and analytical chemistry, as well as the development prospect and analysis of computer in the field of pharmacy. In undergraduate and graduate course teaching occupies an important position.

\section{ACKNOWLEDGMENT}

This study was financially supported by the National Science Foundation of China (No. 21346006), Department of scientific research project in Heilongjiang province (No. B2017015), Scientific research project of Heilongjiang provincial education department (No. 12541783), National project training project of Jiamusi University (No. JMSUJCGP 2016-003), The leader of academic research and education, 2017 Jiamusi University education research hundred works.

\section{REFERENCES}

[1] Wei Fuxiang. Instrument Analysis and Application [M]. Beijing: Sinopec Press, 2007: 71-115. (In Chinese)

[2] Shen Zhaoming, Zhang Yanwei, Liu Zhihong. Application of Computer in Domestic Atomic Absorption Spectrometry Instrument [J]. 1984(4):61-65. (In Chinese)
[3] Zhou Xiuxia. Application of Computer in Chemical Analysis Instrument and Laboratory Management[J]. Metallurgical Analysis, 2000, 20(2): 40-41. (In Chinese)

[4] Jiang Xuehui. Research on key technologies for data processing of chromatography-mass spectrometry[D]. Tianjin University, 2013. (In Chinese)

[5] Wang Ping. On the application of intelligent automation in instrumentation[J]. Heilongjiang Science and Technology Information, 2009, 3:15. (In Chinese)

[6] Wang Shouxu. Research progress in the application of computer technology in chemistry[J]. Heilongjiang Science and Technology Information, 2000, 2(12):119-124. (In Chinese)

[7] An Dengkui. Drug Analysis (3rd Edition), People's Medical Publishing House, 1996:288. (In Chinese)

[8] Shen Hong. Application of computer in hospital pharmacy[J]. Anhui Medical and Pharmaceutical Journal, 2005(02):26-245. (In Chinese)

[9] Gao Shouguo. Design of Database System for Drug Chromato -graphic Separation Conditions. Progress in Pharmacy, 1990, 23(5): 287. (In Chinese)

[10] Li Lingjuan, Hong Hong, Xu Xuesong, Guo Lijun. Application of computer chemistry and its application in the separation of traditional Chinese medicines[J]. China Journal of Chinese Materia Medica, 2011, 36(24):3389-3396. (In Chinese)

[11] Ji Hong, Li Jing. Application of computer in pharmaceutical field[J]. Capital Medical Journal. 2005(24): 24-326. (In Chinese)

[12] Yang zhangwei, Hu Jinhong, Wang Zhuo, Xu Huixin. Design and Application of Computer Software for Clinical Pharmacy Information Service System[J]. Chinese Journal of Clinical Pharmacy, 2003(04):240243. (In Chinese)

[13] Shen Hong. Application of computer in hospital pharmacy[J]. Anhui Medical and Pharmaceutical Journal, 2005(02):26-245. (In Chinese)

[14] Xu Yuchun, Song Yi. Design of infusion monitor[J]. China Medical Devices Information, 2017, 23(07):91-93. (In Chinese)

[15] Huang Hailiang, Li Zhongli, Zhao Dongyong. Application and discussion of computer network technology in hospital pharmacy service [J]. Chinese contemporary medicine, 2011, 18(01):141:27. (In Chinese) 